1. Metadata, including a data sharing plan, should be shared at trial registration.

2. Summary level results and lay summaries should be shared 12 months after study completion.

3. Post-publication data package should be shared within six months of publication.

4. If the regulatory agency does not approve the product, the full data package should be shared 18 months after study completion.

5. If regulatory agency approves the product, a post-regulatory data package should be shared 18 months after product abandonment or 30 days after regulatory approval.

Lay summaries are an important new component that will fulfill the need to disclose information to clinical trial participants, said Lo. "We heard very eloquent testimony from clinical trial participants that they enrolled in a trial and never found out what the investigators found, and we think they need to know what their contributions led to in terms of scientific knowledge."

\section{Ensuring transparency}

The report's third recommendation aims to ensure that data sharing happens fairly. It encourages researchers, sponsors or organizations that hold clinical trial data to implement data-use agreements with other researchers who wish to use the data. In addition, the IOM recommends creating an independent review panel (including public members) that will determine who is able to access the clinical trial data, thereby making the process more transparent.

This recommendation, Lo said, also deals with preventing conflicts of interest when it comes to requesting clinical trial data. "It should not be the company or the principal investigators alone deciding who gets access and who doesn't. It should be an independent neutral party."

Dr. Elizabeth Asztalos, director of clinical trial services at Sunnybrook Research Institute in Toronto, says a major concern is acknowledging who owns the clinical trial data. "The reality is that we have to be very careful as who is defined as owning the data. It's not just the investigators that own the data. Right now, the framework is not there to share data."

"I don't think anyone has any problems sharing data, but we have to recognize the legalities behind it. I can voluntarily want to share data from a trial that I did, but if we didn't do it under the proper legal framework, then we'd get our hands slapped."

\section{Improving infrastructure}

The fourth recommendation concerns the practical challenges of accumulating data in an organized and accessible way. It urges the creation of a multistakeholder body with global reach and a technological enhancement.

Although websites like clinicaltrials. gov, which has 180000 registered studies worldwide, Lo says a lack of a universal and interoperable database is a major problem. "One company may have a way of storing the data and organizing it that is not compatible with how another company does it. If you want to compare two different trials, that's a real headache."

Although newer investigators are embracing a mindset of data sharing, this report is not the end of discussion, said Dr. Ida Sim, professor of medicine at the University of California, and IOM committee member.

"We do need a culture change. For those of us who have been around a little longer, this is a little bit new and I think it's going to take time to transition. The infrastructure, the workforce training - that starts with culture change - I do believe it is starting, and our report is an attempt to move this along." — Dane Wanniarachige, CMAJ

CMAJ 2015. DOI:10.1503/cmaj.109-4980

\title{
Exploring physician compensation
}

$\mathrm{W}$ hen interviewing celebrities on the podcast Comedy Bang! Bang!, host Scott Aukerman sometimes starts with a rather direct question: "So, how much do you make?" He does this because the question is obviously inappropriate and makes for an awkward (and funny) start to the show, as the star attempts to steer the conversation in another direction.

Personal income is, of course, a personal matter, and the subject is no less touchy in the medical profession than in any other occupation. Though physicians would likely rather discuss how to improve patient care than how much money they make, doctors are nonetheless working professionals, not volunteers, and caring about compensation is no crime.
"It's perfectly fair that physicians have an interest in their income. Everybody does," says Jeremiah Hurley, the chair of economics at McMaster University and a member of the Centre for Health Economics and Policy Analysis.

In most societies, doctors are held in high regard and, in general, are fairly well compensated for their work. In a universal health care system, such as Canada's, physician compensation is a major health care expenditure. How physicians are paid, and how much, can also affect clinical care.

"If you put the incentives in the right place, you can significantly change the way medical practice goes," says Dr. David Attwell, a family physician in Victoria and president of the statutory negotiating committee for Doctors of BC.
$C M A J$ will be exploring physician compensation in a series of articles, touching on issues such as:

Government-physician negotiations: What leverage does each side possess in the secretive and often contentious contract negotiations between provincial governments and physician associations? Do the nature of the negotiations change during times of fiscal restraint? Are there unique elements in this process compared to negotiations between government and other professions? Are governments becoming more involved in influencing individual fees for services rather than only negotiating an overall amount? Is this an appropriate role? 


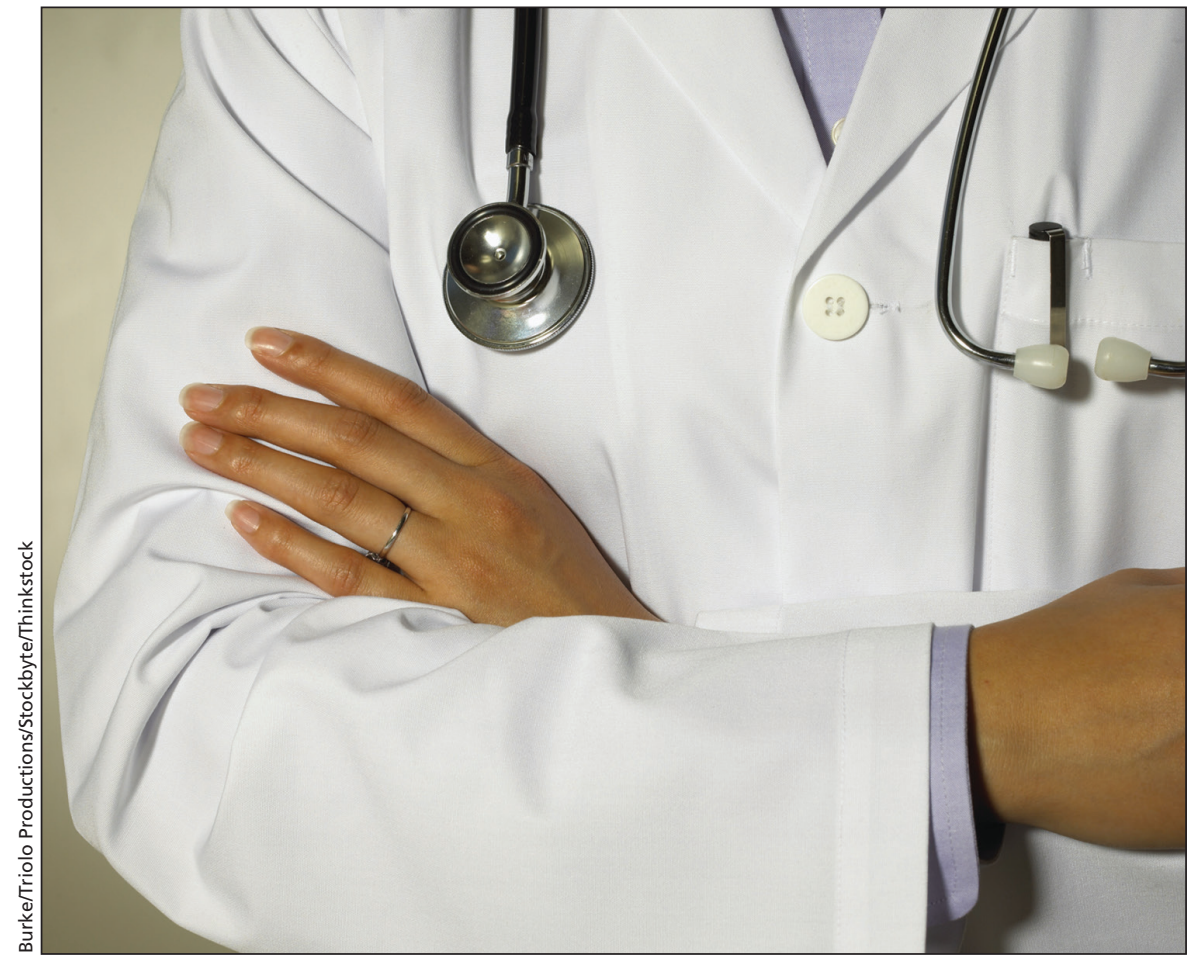

In most societies, doctors are fairly well compensated for their work.

Internal allocation: How is money divided between family medicine and other specialties? How do the specialties allocate funds among themselves? How are funding disparities addressed?

Fair compensation: How much money should a doctor make? How does physician income in Canada compare with other countries? How does the increase in physician compensation in recent decades compare to that of other workers in Canada? Are some specialists earning too much at the expense of other physicians? Does this affect the quality or quantity of clinical services available to Canadians? What is the public's perception of how much doctors make?

Cost of making a doctor: What is the average age and debt load of a new doctor? How many years does it take to pay off that debt? How does illness or pregnancy affect their income? How does the average physician fair in retirement?

Setting a fee: What factors are involved in establishing a fee for a medical service? Are some fees set too low and others too high? Does this affect medial practice? How difficult is it to change a fee once it has been set?
Health care expenditure: How much of the money spent on health care in Canada goes to physicians? Is this figure growing or shrinking relative to other expenditures? Is the amount Canada spends on physician services sustainable?

Geographical variation: Why are doctors paid more in some provinces than others? Do urban physicians earn more than their colleagues in rural areas? How much does overhead vary between a medical practice in a large city and one in a small community? Do some jurisdictions offer better financial incentives than others?

Transparency: Should the billings of individual physicians be publicly disclosed in every province? What are the pros and cons of transparency of gross income? What effects have the publication of this information had in Manitoba and British Columbia?

Other income sources: How common is it for doctors to receive money from sources outside medicare, such as pharmaceutical companies or makers of medical devices? Are these conflicts of interest? Will Canadian companies eventually have to disclose their finan- cial ties to doctors, as is required in the United States, the United Kingdom and other countries?

Overhead: How much do overhead costs vary among medical specialties? Are there studies of overhead that provide precise figures or are there advantages to keeping the percentages vague? Do physicians have the business skills to control overhead and run efficient practices?

Technology: Can new technologies increase physician compensation? Should physicians receive more compensation to offset the costs of adopting new technologies? If technological advances allow medical procedures to be performed in less time, should the fees for those services be reduced?

Business training: Do medical students receive adequate education on the nonclinical aspects of running a successful medical practice? Could medical schools learn something about providing business training from other health education institutions, such as dental schools? Or is it the job of other institutions, such as teaching hospitals or accrediting bodies such as the medical colleges, to provide this training?

Selling products or services: Is the line between clinician and salesperson blurred when doctors offer to sell products (e.g., skin creams, vitamins) or services (e.g., laser eye surgery, cosmetic lesion removal) to their patients?

Alternative payment models: Do physicians earn more outside the feefor-service system? What financial incentives are provided in alternative payment models and do they affect patient care?

Pay and prestige: Would the status of the medical profession be lessened if incomes were lower? Is the potential for high pay a reason people choose medicine for a career? Would medical schools have trouble attracting bright students if doctors weren't paid well? - Roger Collier, CMAJ

CMAJ 2015. DOI:10.1503/cmaj.109-4974 